\title{
Plasmacytoid Urothelial Carcinoma: Response to Chemotherapy and Oncologic Outcomes
}

Leonidas N. Diamantopoulos ${ }^{\mathrm{a}}$, Ali Raza Khaki ${ }^{\mathrm{a}}$, Petros Grivas ${ }^{\mathrm{a}, \mathrm{b}}$, John L. Gore ${ }^{\mathrm{c}}$, George R. Schade ${ }^{\mathrm{c}}$, Andrew C. Hsieh ${ }^{\mathrm{a}, \mathrm{b}}$, John K. Lee ${ }^{\mathrm{a}, \mathrm{b}}$, Todd Yezefski ${ }^{\mathrm{a}}$, Evan Y. Yu ${ }^{\mathrm{a}, \mathrm{b}}$, Michael T. Schweizer ${ }^{\mathrm{a}, \mathrm{b}}$, Heather H. Cheng ${ }^{\mathrm{a}, \mathrm{b}}$, Sarah P. Psutka ${ }^{\mathrm{c}}$, Daniel W. Lin ${ }^{\mathrm{b}, \mathrm{c}}$, Maria S. Tretiakova ${ }^{\mathrm{d}}$, Funda Vakar-Lopez ${ }^{\mathrm{d}}$, Robert B. Montgomery ${ }^{\mathrm{a}, \mathrm{b}}$ and Jonathan L. Wright ${ }^{\mathrm{b}, \mathrm{c}, *}$

${ }^{a}$ Department of Medicine, Division of Medical Oncology, University of Washington, Seattle Cancer Care Alliance, Seattle, WA, USA

${ }^{\mathrm{b}}$ Fred Hutchinson Cancer Research Center, Seattle, WA, USA

${ }^{\mathrm{c}}$ Department of Urology, University of Washington, Seattle, WA, USA

${ }^{\mathrm{d}}$ Department of Pathology, University of Washington, Seattle, WA, USA

Received 12 November 2019

Accepted 27 January 2020

\begin{abstract}
.
BACKGROUND: Plasmacytoid urothelial carcinoma is a rare bladder cancer variant with scarce data on outcomes and prognostic factors.

OBJECTIVE: We report our institutional experience with this histology to determine response to neoadjuvant chemotherapy, definitive surgery and survival.

METHODS: We conducted a retrospective chart review of consecutive patients with plasmacytoid, as well as conventional urothelial carcinoma (for comparison) seen in our institution (2007-2018). Baseline characteristics, clinicopathologic and treatment data were captured. $T$-test, chi-squared and log-rank test was used for group comparison. Kaplan Meier method was used for estimation of overall survival and Cox regression for identification of prognostic factors.

RESULTS: 64 patients with plasmacytoid and 418 with conventional urothelial histology were identified; $53 \%$ of those with plasmacytoid presented with cT3/4 stage and 67\% underwent extirpative surgery. Patients with plasmacytoid histology had higher rates of pT3/4 (65\% vs. $28 \%$ ), nodal disease (37\% vs. 16\%) and positive surgical margins (23\% vs. 5\%) compared to urothelial group $(p<0.01)$, as well as higher incidence of post-operative recurrence $(47 \%$ vs. $29 \%, p=0.05)$ and lower ypTON0 rates after neoadjuvant chemotherapy $(10 \%$ vs. $33 \%, p=0.03)$. Plasmacytoid histology was associated with lower median overall survival compared to conventional urothelial (24 vs. 154 months, $p<0.01$ ).

CONCLUSIONS: Plasmacytoid urothelial carcinoma frequently presented with advanced stage at diagnosis and extirpative surgery, poor pathologic response to neoadjuvant chemotherapy, and inferior outcomes, when compared to conventional urothelial. Prospective trials evaluating upfront cystectomy versus preoperative chemotherapy and/or novel treatments should be considered.
\end{abstract}

Keywords: Urinary bladder neoplasms, transitional cell carcinoma, neoadjuvant therapy, cystectomy

\footnotetext{
${ }^{*}$ Correspondence to: Jonathan L. Wright, MD, MS, FACS, Associate Professor of Urology, University of Washington School of Medicine, 1959 NE Pacific Box 356510, Seattle, WA 98195,
}

USA. Tel.: +1 2065434740; Fax: +1 206543 3272; E-mail: jlwright@uw.edu. 


\section{INTRODUCTION}

Plasmacytoid urothelial carcinoma (PUC) is a rare urothelial carcinoma (UC) variant with an aggressive phenotype [1], comprising approximately 3-5\% of patients with muscle-invasive bladder cancer. Histologically, PUC can appear as diffuse sheets of cells or as a discohesive, single-cell and highly infiltrative growth pattern. The cells with their eccentrically located nuclei and a moderate-toabundant eosinophilic cytoplasm, resemble plasma cells [2], Fig. 1. Molecularly and histologically, PUC is characterized by loss of cytokeratin 20, a high proliferation index, tumor protein 53 accumulation, and loss of membranous E-cadherin expression [3]. In terms of clinical behavior, case series have reported that patients with PUC more commonly present with advanced stage [2], positive margins at radical cystectomy (RC) [4], locoregional spread along fascial planes [5] and propensity for peritoneal carcinomatosis [6].

The standard of care for muscle-invasive UC is cisplatin-based neoadjuvant chemotherapy (NAC) with either accelerated (dose dense) methotrexate/vinblastine/adriamycin/cisplatin (MVAC) or gemcitabine/cisplatin (GC) [7, 8]. In general, PUC has been consistently associated with poor prognosis despite the use of standard bladder cancer treatment modalities, based on retrospective data across the literature [3]. In addition, applying the NAC paradigm to PUC is based on scarce and equivocal data to date, mainly extrapolating from conventional UC [9, 10], while the question of appropriate management in those patients remains unanswered. The purpose of this study was to elucidate clinicopathologic factors related to PUC and investigate response to NAC, also in relation to conventional UC. We hypothesized that patients with PUC would present with more advanced stage at diagnosis and at $\mathrm{RC}$ (vs. conventional UC), and that NAC would not significantly influence pathologic stage or overall survival (OS), in comparison with upfront RC.

\section{MATERIALS AND METHODS}

After obtaining institutional review board approval (IRB\# STUDY00005690), we retrospectively reviewed the charts of all consecutive patients with PUC treated at our institution from January 2007 to December 2018, based on pathology review [transurethral resection of bladder tumor (TURBT) and/or definitive surgery] by institutional experts. In compliance with the IRB regulations we obtained waiver of consent, also considering the retrospective and observational nature of the study. No prospective human experimentation was involved.

We included all cases with plasmacytoid features, regardless of the percentage of plasmacytoid histology.

In addition, we retrospectively assessed our institutional RC database, to identify consecutive patients only with conventional (pure) UC in both TURBT and RC specimens (no variant histology present). All cases were reviewed by an expert pathologist with significant experience in genitourinary cancers. Since a small number of PUC cases were treated with definitive surgery other than RC (one partial cystectomy and one segmental ureterectomy), we utilized the umbrella term "extirpative surgery" to refer to all types of definitive surgery. We collected demographic data, baseline clinicopathologic data [clinical stage at diagnosis, pathologic stage at surgery, pathologic variants with percentages, presence of lymphovascular invasion (LVI) and carcinoma in situ (CIS)], preoperative laboratory workup, baseline historical data, medical comorbidities and treatment modalities [TURBT, intravesical bacillus Calmette-Guerin (BCG), surgery, neoadjuvant / adjuvant chemotherapy, chemoradiation, palliative chemotherapy and/or radiation regimens]. In terms of NAC, we captured the regimens, number of cycles received, first and last date of infusion, clinically significant complications [deep venous thrombosis/pulmonary embolism (DVT/PE), neutropenia (including febrile), acute kidney injury (AKI), need for hospital admission during NAC receipt] radiographic and pathologic response. Pathologic complete response to NAC was indicated as ypTONO.

Statistical analysis was performed with STATA package (College Station, Texas). T-tests and chisquared tests (Pearson, Fischer's exact test) were used for group comparison analysis. Kaplan-Meier curves were created for median overall survival (OS) estimation, measured from the date of cancer diagnosis to all-cause mortality in the entire cohort, and from the date of extirpative surgery in the surgical subgroup. Median recurrence free survival (RFS) from the time of extirpative surgery to initial radiographic/pathologic recurrence or death was estimated with the same method. Log-rank test was used for unadjusted comparisons of OS and RFS between the groups. 5-year OS and RFS cumulative rates (with standard error reported in parentheses) 

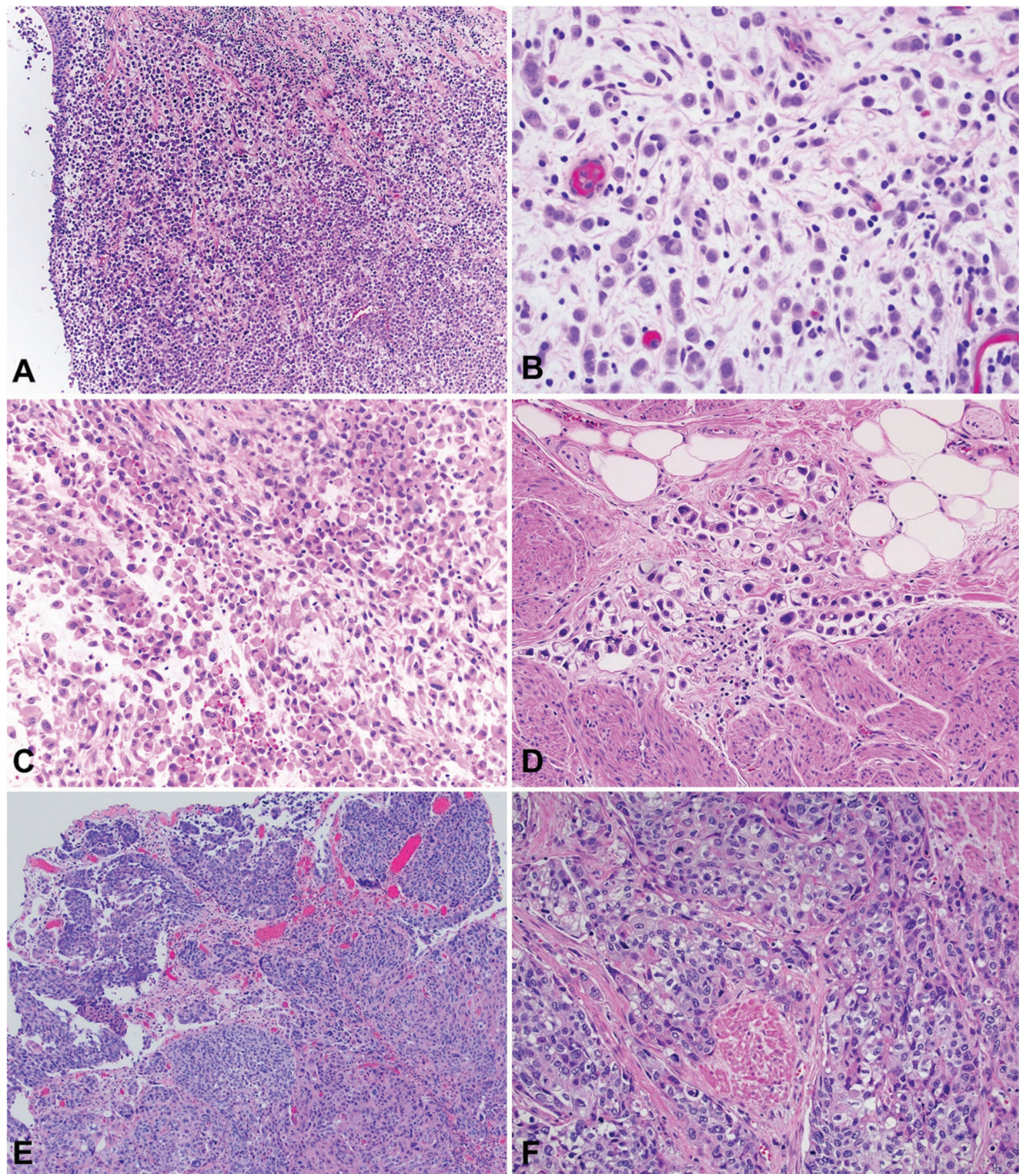

Fig. 1. Photomicrography of bladder tumors. A - Plasmacytoid urothelial carcinoma (40x). B - Plasmacytoid urothelial carcinoma (200x). C - Plasmacytoid urothelial carcinoma with rhabdoid features (100x). D - Plasmacytoid urothelial carcinoma with signet ring features (200x). E - Conventional urothelial carcinoma (40x). F - Conventional urothelial carcinoma with muscle invasion (100x).

were estimated with life-table analysis. Multivariate Cox-regression analysis was performed for OS. The primary predictor of interest was the use of NAC. Covariates tested were age at diagnosis, gender, cT stage, adjuvant therapy, pT, pN stage, positive surgi- cal margins, extravesical disease and the presence of CIS and LVI status at extirpative surgery. Covariates were included in the final multivariate Cox-regression model if they changed the hazard ratio (HR) for NAC by $>10 \%$ from the base model. A $5 \%$ alpha 
error was considered as the cutoff of statistical significance.

\section{RESULTS}

\section{Baseline demographic/diagnostic data}

64 consecutive patients with PUC and 418 with conventional UC were identified from the same time period. Baseline demographics and clinicopathologic data of patients with PUC and UC are documented in Table 1. PUC component was present at TURBT for 50 patients and only found at extirpative surgery for 14 patients.

\section{Treatment summary}

\section{Surgical group}

Extirpative surgery (RC, partial cystectomy or segmental ureterectomy) was performed in 43/64 (67\%) patients with PUC. At presentation, 20/43 (47\%) had clinical suspicion of disease beyond muscularis propria (cT3/4). At the time of surgery, 28/43 (65\%) patients with extravesical disease (pT3/4) were eventually identified, and upstaging occurred in 19/43 (44\%). CIS and LVI were present in 15/43 (35\%) and 24/43 (56\%), respectively, whereas 10/43 (23\%) had positive surgical margins and 16/43 (37\%) had $\mathrm{pN}+$ stage. One or more complications in the immediate post-operative period were noted in 8/43 (19\%) patients. Post-surgical localized or distant recurrence was documented in 20/43 (47\%) patients. In chisquared test (Table 2), the incidence of cT3/4, pT3/4, $\mathrm{pN}+$ disease, LVI, positive surgical margins and postoperative recurrence was significantly lower in the conventional UC group, while pTON0 rate was significantly higher. Also, among sites of postoperative recurrence, peritoneal involvement was noted at a significantly higher proportion in patients with PUC, in comparison with conventional UC. At the same time, there were no significant differences in baseline characteristics between the two cohorts, in terms of age and gender.

\section{Neoadjuvant chemotherapy}

NAC was offered to $166 / 418$ patients (40\%) in the UC and 26/64 patients (41\%) in the PUC cohort. In the latter, 21/64 patients (33\%) underwent RC, 2/64 (3\%) were switched to salvage chemotherapy after progressing on NAC, 2/64 (3\%) elected for bladder sparing chemoradiotherapy despite radiographic response/stable disease on NAC, and 1/64
Table 1

Baseline demographic and treatment data for Plasmacytoid (PUC) and conventional (pure) Urothelial Carcinoma (UC) cohorts

\begin{tabular}{|c|c|c|}
\hline & PUC $(n=64)^{*}$ & $\mathrm{UC}(n=418)$ \\
\hline Variables & $\mathrm{n}(\%)$ & $\mathrm{n}(\%)$ \\
\hline \multicolumn{3}{|l|}{ Gender } \\
\hline male & $52(81)$ & $332(79)$ \\
\hline female & $12(19)$ & $86(21)$ \\
\hline \multicolumn{3}{|l|}{ Race } \\
\hline Caucasian & $58(91)$ & $382(91)$ \\
\hline African American & $1(2)$ & $8(2)$ \\
\hline Asian & $5(8)$ & $11(3)$ \\
\hline Hispanic & $0(0)$ & $4(1)$ \\
\hline$N / A$ & $0(0)$ & $13(3)$ \\
\hline Median age (years, IQR) & $67(62-75)$ & $67(59-75)$ \\
\hline \multicolumn{3}{|l|}{ Smoking History } \\
\hline Past & $32(50)$ & $207(50)$ \\
\hline Active & $16(25)$ & $93(22)$ \\
\hline Never & $16(25)$ & $118(28)$ \\
\hline \multicolumn{3}{|l|}{ Performance Status } \\
\hline$E C O G 0-1$ & $52(81)$ & $387(93)$ \\
\hline$E C O G \geq 2$ & $12(19)$ & $31(7)$ \\
\hline \multicolumn{3}{|l|}{ Tumor Site } \\
\hline Bladder & $61(95)$ & $418(100)$ \\
\hline Upper tract & $2(3)$ & $0(0)$ \\
\hline Urethra & $1(2)$ & $0(0)$ \\
\hline \multicolumn{3}{|l|}{ Clinical stage at diagnosis } \\
\hline$c T x$ & $1(2)$ & $0(0)$ \\
\hline$c T a$ & $0(0)$ & $25(6)$ \\
\hline cTis & $0(0)$ & $22(5)$ \\
\hline$c T 1$ & $11(17)$ & $82(20)$ \\
\hline$c T 2$ & $18(28)$ & $162(39)$ \\
\hline cT3/T4 & $34(53)$ & $127(30)$ \\
\hline$c N 1-3$ & $10(16)$ & $29(7)$ \\
\hline$M 1 a / b$ & $20(31)$ & $6(1)$ \\
\hline \multicolumn{3}{|l|}{ Extirpative Surgery } \\
\hline Radical cystectomy & $41(64)$ & $418(100)$ \\
\hline Partial cystectomy & $1(2)$ & - \\
\hline Segmental ureterectomy & $1(2)$ & - \\
\hline \multicolumn{3}{|l|}{ Perioperative chemotherapy } \\
\hline Neoadjuvant & $26(41)$ & $166(40)$ \\
\hline Adjuvant & $15(23)$ & $22(5)$ \\
\hline
\end{tabular}

*Includes all PUC cases regardless of whether extirpative surgery was performed. Abbreviations: IQR-interquartile range, ECOGEastern Cooperative Oncology Group, c- clinical, T - tumor stage, $\mathrm{N}$ - nodal stage, $\mathrm{M}$ - metastasis, N/A - not available.

(2\%) had progression of a second primary tumor (non-small cell lung cancer) and was treated in the palliative setting. All patients with NAC received at least one dose of MVAC (dose dense) or GC. Clinically significant complications from NAC were noted in $12 / 26(46 \%)$ patients, including neutropenia (5), AKI (4) and DVT/PE (3), leading to hospitalization in three cases. Radiographic progression during NAC was noted in 6/26 (23\%) patients; two were switched to palliative chemotherapy and four proceeded to salvage cystectomy. At definitive surgery, $3 / 21$ (14\%) had pathologic response; $2 / 21$ (10\%) had complete response (ypT0N0), 1/21 (5\%) had 
Table 2

Clinicopathologic characteristics of patients with Plasmacytoid (PUC) and conventional (pure) Urothelial Carcinoma (UC) treated with extirpative surgery

\begin{tabular}{|c|c|c|c|}
\hline Variables & $\begin{array}{c}\mathrm{PUC}(n=43) \\
\mathrm{n}(\%)\end{array}$ & $\begin{array}{c}\mathrm{UC}(n=418) \\
\mathrm{n}(\%)\end{array}$ & $\begin{array}{c}P \text { value (Pearson } \chi^{2}, \\
\text { Fischer exact test, } t \text {-test) }\end{array}$ \\
\hline Gender & & & 0.96 \\
\hline male & $34(79)$ & $332(79)$ & \\
\hline female & $9(21)$ & $86(21)$ & \\
\hline Median age (years, IQR) & $65(59-71)$ & $67(59-75)$ & 0.87 \\
\hline Clinical stage at diagnosis & & & 0.09 \\
\hline$<c T 2$ & $9(21)$ & $129(31)$ & \\
\hline$c T 2$ & $14(33)$ & $162(39)$ & \\
\hline$c T 3 / 4$ & $20(47)$ & $127(30)$ & 0.03 \\
\hline Nodal disease (imaging) & $4(9)$ & $29(7)$ & 0.57 \\
\hline$C I S+(T U R B T)$ & $13(30)$ & $126(30)$ & 0.92 \\
\hline$L V I+(T U R B T)$ & $8(19)$ & $40(10)$ & 0.06 \\
\hline Pathologic stage (ES) & & & $<0.01$ \\
\hline$<p T 2$ & $7(16)$ & $241(58)$ & \\
\hline$p T 2$ & $8(19)$ & $62(15)$ & \\
\hline$p T 3 / T 4$ & $28(65)$ & $115(28)$ & \\
\hline Nodal disease (ES) & $16(37)$ & $67(16)$ & $<0.01$ \\
\hline pTONO & $4(9)$ & $96(23)$ & 0.03 \\
\hline Positive margins (ES) & $10(23)$ & $21(5)$ & $<0.01$ \\
\hline$C I S+(E S)$ & $15(35)$ & $101(24)$ & 0.12 \\
\hline$L V I+(E S)$ & $24(56)$ & $68(16)$ & $<0.01$ \\
\hline Postoperative recurrence* & $n=20(47)$ & $n=122(29)$ & 0.05 \\
\hline Pelvis/pelvic LNs & $6(30)$ & $36(30)$ & 0.96 \\
\hline Liver & $3(15)$ & $25(20)$ & 0.77 \\
\hline Peritoneal & $6(30)$ & $6(5)$ & $<0.01$ \\
\hline Lungs & $1(5)$ & $33(27)$ & 0.04 \\
\hline Bones & $8(40)$ & $27(22)$ & 0.10 \\
\hline Brain & $3(15)$ & $6(5)$ & 0.12 \\
\hline
\end{tabular}

*Recurrence sites are not mutually exclusive, total number of recurrent cases in each group was used as denominator. Abbreviations: IQR - interquartile range, TURBT - transurethral resection of bladder tumor, $\mathrm{p}$ - pathologic, c- clinical, $\mathrm{T}$ - tumor stage, $\mathrm{N}$ - nodal stage, CIS - carcinoma in situ, LVI - lymphovascular invasion, ES - extirpative surgery, LN - lymph nodes.

partial response (ypT1N0). Upstaging occurred in $7 / 21(33 \%)$ patients. Four patients treated with NAC had no residual PUC histology in the cystectomy specimen, while there was still ypT2/3 UC present. Conversely, four NAC-treated patients had no PUC at TURBT, but PUC component was found at RC.

Each histology subgroup received a median of four NAC cycles. In the PUC subgroup, using chi squared test, no significant association was identified between NAC and pT0N0 $(p=0.96)$, extravesical disease at surgery, $(p=0.84), \mathrm{pN}+$ status $(p=0.72)$, margin positivity $(p=0.93), \mathrm{LVI}+(p=0.33)$ or CIS+ $(p=0.14)$. Likewise, NAC was not associated with improved OS (26.0 vs. 23.6 months, $p=0.36$ ), when compared to upfront extirpative surgery. In comparison, in the conventional UC group, patients who received NAC had significantly higher rates of pTONO $(p<0.01)$ and lower incidence of extravesical disease $(p=0.02), \mathrm{CIS}+(p<0.01)$ and LVI+ $(p=0.05)$ rates, but no notable difference in $\mathrm{pN}+(p=0.89)$ and positive surgical margins ( $p=0.44$ ), compared to extirpative surgery alone. The incidence of ypT0NO after NAC receipt was significantly higher in the conventional UC vs. PUC group [54/166 (33\%) vs. $2 / 21$ (10\%), $p=0.03$ ].

\section{Non-surgical group}

Overall, 20/64 (31\%) patients were not treated with extirpative surgery for PUC, due to unresectable/metastatic disease at diagnosis (8), death shortly after diagnosis (4), poor functional status (2), election for bladder-sparing chemoradiotherapy (2), progressive disease during NAC (2) or BCG treatment (1), progression of another primary (1). In addition, one patient was lost to follow-up after initial diagnosis and treatment data was not available. Among patients presenting with unresectable/metastatic disease (8), primary cisplatin-based palliative chemotherapy was offered 


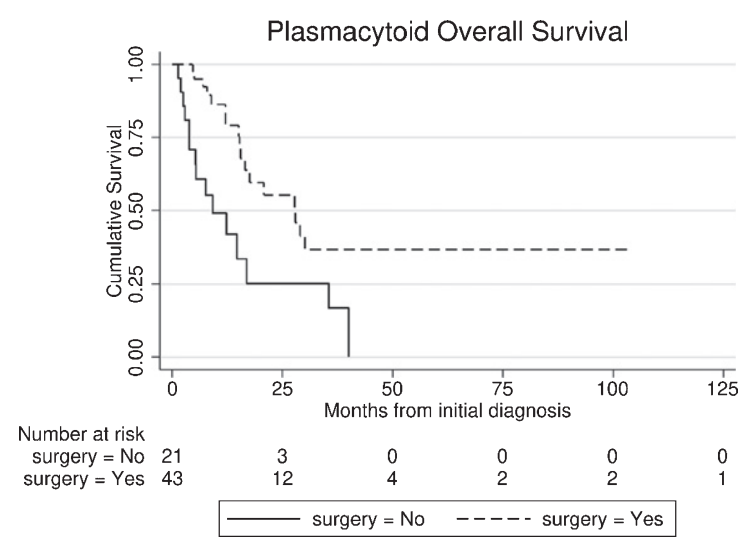

Fig. 2. Overall survival (OS) from the time of diagnosis of plasmacytoid urothelial carcinoma (PUC), between surgical and non-surgical group.

to four, targeted therapy as part of a clinical trial to one, concurrent chemoradiotherapy to two, and pelvic radiotherapy-only to one. Subsequent treatment lines for patients who progressed on first-line palliative chemotherapy or NAC, were: pemetrexed (2), pemetrexed/docetaxel (1), gemcitabine/carboplatin followed by pembrolizumab (1), pembrolizumab alone (1). Also, GC, followed by gemcitabine/carboplatin due to intolerance, was offered to a patient who progressed to pelvis and retroperitoneal lymph nodes after BCG treatment for T1 bladder tumor. Four patients died shortly after diagnosis, without receiving any treatment, due to complications from rapidly progressive disease (1), stroke and aspiration pneumonia (1), sternal wound infection after chondrosarcoma resection (1), clinical deterioration due to disease-related complications (1).

\section{Survival analysis and prognostic factors}

In the entire PUC cohort, including surgical and non-surgical patients $(n=64)$, median follow-up was 34.1 months [95\% Confidence Interval (CI): 10.1 58.0], while 32/64 (50\%) deaths were noted (27 bladder cancer - related). Median OS was 17.6 months (95\% CI: 4.6 - 30.6) from PUC diagnosis. Patients who underwent extirpative surgery had superior OS (from diagnosis) compared with the non-surgical group (27.9 vs. 9.2 months, $p<0.01$ ), Fig. 2.

Median OS (from the time of extirpative surgery) in the surgical PUC subgroup was significantly inferior to conventional (pure) UC (23.6 vs. 154.0 months, $p<0.01$, Fig. 3), and the same was true for median RFS (13.7 vs. 99.4 months, $p<0.01)$. Five-year

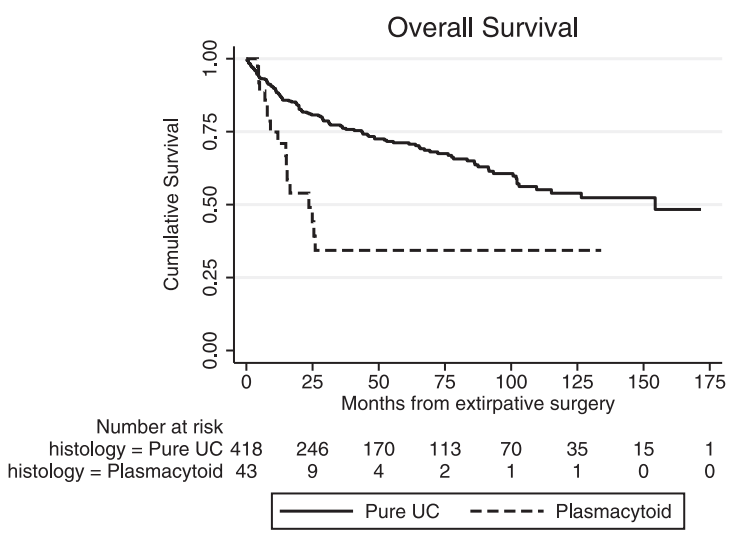

Fig. 3. Overall survival (OS) from the time of extirpative surgery for conventional (pure) urothelial carcinoma (UC) and plasmacytoid UC (PUC).

cumulative OS was $35 \%( \pm 10)$ in patients with PUC and $71 \%( \pm 3)$ in those with conventional UC, while five-year cumulative RFS was $33 \%( \pm 9)$ and $60 \%( \pm 3)$, respectively. In univariate Cox regression for OS in the entire surgical cohort (UC and PUC, $n=461$ ), presence of plasmacytoid variant was significantly associated with worse OS (HR 2.78, 95\% CI: $1.65-4.64)$. However, in the multivariate model, plasmacytoid histology was no longer significant, with $\mathrm{pT}$ stage, $\mathrm{pN}+$ and advanced age at surgery being the primary determinants of OS, Table 3. Additionally, when we stratified OS analysis by pT stage at $\mathrm{RC}$, no significant differences between the PUC and the UC subgroup were noted for non-muscle invasive disease $(<\mathrm{pT} 1, p=0.48)$ and extravesical disease (pT3/4, $p=0.28$ ), while there was a marginal difference in OS for those with muscle invasive tumors confined to bladder (pT2, $p=0.04$ ), in favor of conventional UC. Similar results were yielded when we compared the plasmacytoid surgical subgroup with conventional UC patients that presented with cT2-4 disease only $(n=289)$. In overall unadjusted comparison, PUC had a significantly inferior median OS $(p<0.001)$ but in stratified analysis, this effect was primarily attributed to the pT2 subgroup $(p<0.01)$, while no significant differences were noted for $\mathrm{pT} 1$ $(p=0.58)$ and $\mathrm{pT} 3 / 4(p=0.19)$ stages.

\section{DISCUSSION}

In this study, we present outcomes from a single institutional cohort of 64 patients with PUC. A significant finding was the low ypTON0 rate $(10 \%)$ after cisplatin-based NAC, which was inferior to 
Table 3

Multivariate Cox Regression for prognostic factors of overall survival (OS) from the time of extirpative surgery

\begin{tabular}{lcc}
\hline $\begin{array}{l}\text { UC and PUC } \\
(\mathrm{N}=461)\end{array}$ & $\begin{array}{c}\text { HR (95\% Confidence } \\
\text { Interval) }\end{array}$ & $p$-value \\
\hline Plasmacytoid component & $1.20(0.62-2.32)$ & 0.58 \\
Gender (female) & $1.27(0.81-2.01)$ & 0.29 \\
Age at Surgery & $1.02(1.01-1.04)$ & 0.03 \\
pT3/4 & $2.18(1.18-4.04)$ & 0.01 \\
pN+ & $2.14(1.33-3.43)$ & $<0.01$ \\
Positive surgical margins & $0.77(0.37-1.6)$ & 0.49 \\
LVI at surgery & $1.56(0.94-2.59)$ & 0.08 \\
Neoadjuvant Chemotherapy & $1.32(0.89-1.94)$ & 0.17 \\
\hline
\end{tabular}

Abbreviations: UC - urothelial carcinoma, PUC - plasmacytoid urothelial carcinoma, HR - Hazard Ratio, pT - pathologic tumor stage, $\mathrm{pN}$ - pathologic nodal stage, LVI - lymphovascular invasion.

conventional UC patients (33\%) over the same time period. In addition, NAC use in PUC patients was not associated with improvements in the presence of extravesical disease, positive surgical margins and $\mathrm{pN}+$ status at the time of surgery, nor with improved OS, compared to upfront RC. These results suggest that NAC use may be less effective in the PUC setting, thus raising the question whether it adds benefit vs. $\mathrm{RC}$ alone in those patients.

In the literature, data on PUC "chemo-sensitivity" is scarce, with variable results reported across studies, Table 4. In one cohort of 31 patients with PUC, NAC was administered to five (stage I-III) patients and it was associated with $80 \%$ pathologic downstaging and $60 \%$ ypT0N0. However, in the same study, NAC was also administered to seven patients with stage IV, followed by salvage cystectomy, but no pathologic response was noted [9]. HashemiShadraei et al. reported poor "chemo-sensitivity" in a subset of 14 patients with PUC, treated with cisplatinbased NAC and RC; one patient had ypT0N0 and $12 / 14(86 \%)$ had persistent extravesical disease at RC [10]. Another study with 33 patients treated with NAC and RC demonstrated 22\% pathologic response (<pT2N0), with $12 \%$ ypT0N0 [11]. Similar results were yielded by Ericson et al. [12]; NAC was administered to 12 patients, but in multivariate analysis, it failed to show a significant effect on pT0 incidence or organ confined disease. Overall, these results are in concordance with our findings, in part validating the observation of low likelihood of PUC pathologic response to NAC. The complete response rate (ypT0N0) in our conventional UC patients over the same time frame is consistent with prior studies $(25-30 \%)[7,8]$.

In terms of clinicopathologic behavior and outcomes, our observations confirmed the aggressive nature of plasmacytoid variant reported previously [4, 5, 9-13]. Patients in our surgical PUC cohort had significantly higher propensity towards advanced stage at diagnosis (47\%) and extravesical disease at surgery (65\%), accompanied by frequently positive nodal status (37\%) and surgical margins (23\%), as well as inferior median OS and RFS, when compared to the conventional UC group. Additionally, there was a significantly higher proportion of patients with postoperative peritoneal recurrence in the PUC group. On the other hand, presence of plasmacytoid histology was not independently associated with worse survival outcomes in multivariate analysis, with advanced age, pT3/4 stage and pN+ disease being the primary negative prognostic factors. This result could be partially attributed to the small proportion of patients with PUC in the entire cohort. In a similar fashion, there were no significant differences in OS when the analysis was adjusted for $\mathrm{pT}$ stage at $\mathrm{RC}$, with the exception of pT2 stage. In general, those findings are suggesting that the worse outcomes in patients with PUC are primarily mediated by well-established factors (age, stage). In the literature, historical data for conventional UC reported lower rates of cT3/4 stage (11\%) at diagnosis and pT3/4 (43\%) stage at RC [14]. Positive surgical margins have a reported incidence of $1-10 \%$ in conventional UC across stages [15], and up to $12 \%$ in cases of extravesical disease extension [16]. Nodal-positive disease at RC can be found in approximately $25 \%$ in general, and up to $34 \%$ in patients with advanced pT stage [16, 17].

The highly infiltrative potential of PUC has been linked to PUC-specific somatic loss-of-function mutations of CDH1, leading to loss of E-cadherin expression, likely promoting cellular detachment and aggressive localized invasion [18]. The discohesive, single-cell growth pattern in histopathology (Fig. 1), along with the increased frequency of localized and peritoneal recurrence (Table 4) are consistent with this finding. E-cadherin loss in 57\% of PUC specimens has also been reported by others, including Perrino et al. [19]. These authors described stratification of PUC into desmoplastic, classic and pleomorphic morphology, with loss of E-cadherin being more prominent in the last two categories, while 9p21 deletion and FGFR3 mutations were frequently identified, providing insight on molecular pathogenesis and potential therapeutic targets. Taking these findings into consideration, expanding our knowledge in the genomic level of metastatic UC and its variants (including PUC) is of paramount importance, with rapid autopsy programs being an interesting 


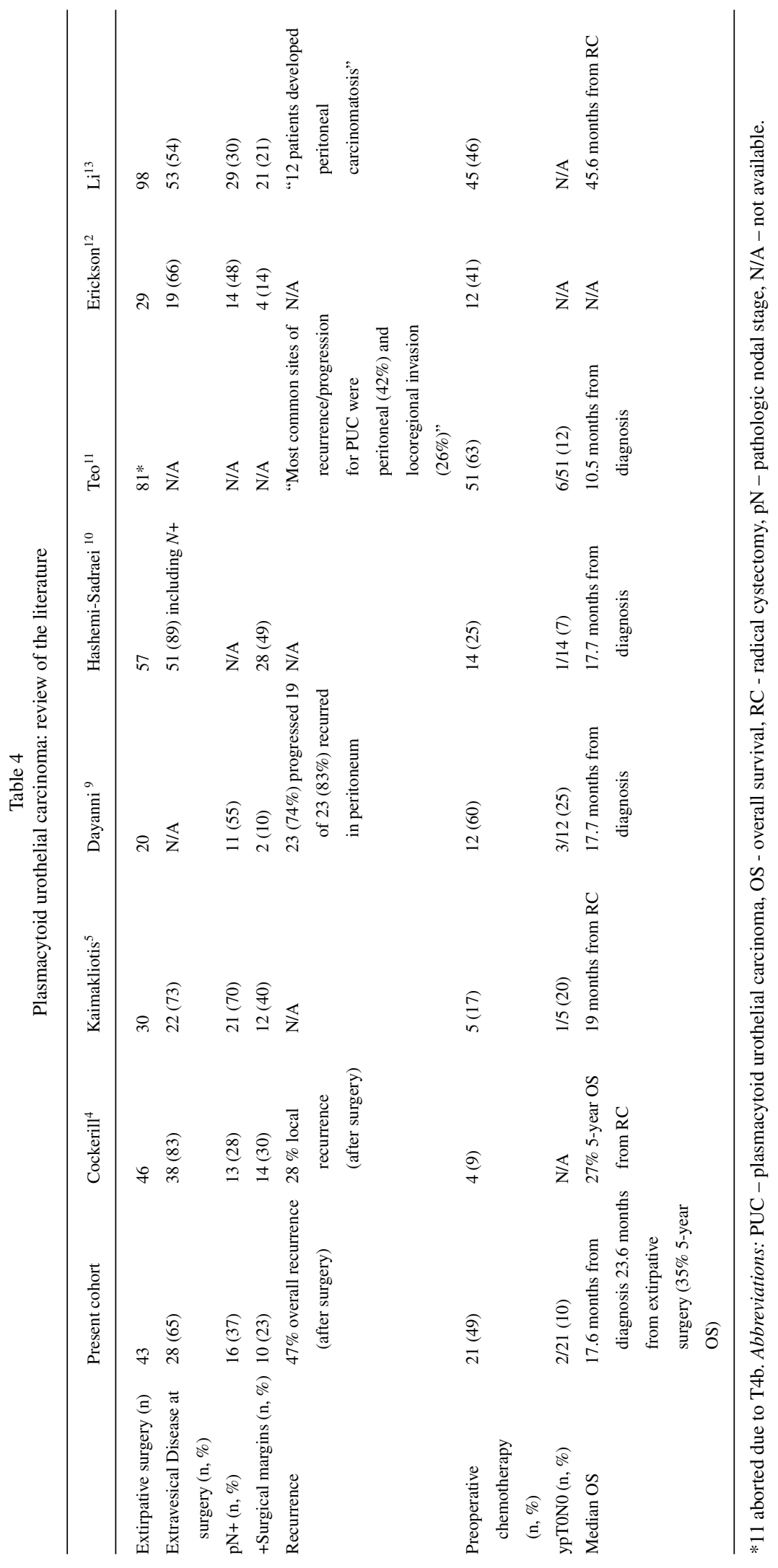


initiative that allows tumor tissue acquisition from multiple sites of metastasis, in the immediate postmortem period [20].

Our PUC cohort is one of the largest reported in the literature, with the additional inclusion of a comparative analysis with conventional UC. However, due to its retrospective nature, our study has a number of limitations, including data acquired from a single institution, small sample size with low number of events, lack of randomization, presence of potential selection and confounding biases, variability in treatment modalities and monitoring/surveillance schedules, lack of biomarker evaluation and of specific \% of plasmacytoid component, as well as loss to follow-up in a number of patients. Patients with advanced - and potentially more aggressive disease, were more likely to receive NAC, which could also influence outcomes independent of NAC. Associations can be deduced but direct causality cannot be inferred. Despite the above, our results are hypothesis-generating, supporting further studies in PUC treatment strategies and evaluation of biomarkers.

\section{CONCLUSIONS}

This institutional study confirms that PUC is an aggressive variant, demonstrating increased propensity for early extravesical spread and lymph node infiltration, when compared with conventional UC. In addition, we observed suboptimal response to NAC, with a $10 \%$ ypT0N0 rate. The low likelihood of complete response or downstaging raises a therapeutic dilemma between NAC and upfront RC. Considering the inconclusive literature on the "chemo-sensitivity" for PUC, our findings underscore the importance of larger dedicated studies on PUC, investigating the rationale of chemotherapy utilization in the perioperative setting, with additional focus on molecular profiling, further genomic interrogation for potential "druggable" targets, and assessment of immunotherapy.

\section{ACKNOWLEDGMENTS}

Dr. Ali R Khaki is supported by the National Cancer Institute under award number T32CA009515, unrelated to this manuscript. We would like to thank Seattle Translational Tumor Research for their support in developing this project.

\section{FUNDING}

The present study did not receive any specific grant from funding agencies in the public, commercial, or not-for-profit sectors.

\section{AUTHOR CONTRIBUTIONS}

All authors contributed in the conception, performance and interpretation of data presented, as well as in the manuscript preparation and approval of the final version.

\section{CONFLICT OF INTEREST}

ARK: Stock and Other Ownership Interests Pfizer, Procter \& Gamble, Walgreens Boots Alliance.

PG: personal fees and other - Genentech, Bayer, Merck \& Co., Mirati Therapeutics, Pfizer, BristolMyers Squibb; personal fees, non-financial support and other - Astra Zeneca, ClovisOncology, EMD Serono; personal fees - Biocept, Seattle Genetics, Foundation Medicine, Driver Inc., QED Therapeutics, Heron Therapeutics, Janssen, GlaxoSmithKline, Roche, Genzyme, Exelixis; other - Oncogenex, Bavarian Nordic, Immunomedics, Debiopharm.

JLG: Research Funding - Ferring Pharmaceuticals.

GRS: Patents, Royalties, Other Intellectual Property - Global Cancer Technology.

ACH: Honoraria-Hotspot Therapeutics; Research Funding - eFFECTOR Inc.; Patents, Royalties, Other Intellectual Property - MTOR modulators and uses thereof Patent number: 9629843; Use of translational profiling to identify target molecules for Therapeutic treatment Publication number: 20140288097.

JKL: Research Funding - Immunomedics.

EYY: Consulting or Advisory Role - Amgen, AstraZeneca, Bayer, Churchill Pharmaceuticals, Dendreon, EMD, Serono, Incyte, Janssen, Merck, QED, Seattle Genetics, Tolmar; Research Funding Agensys, Astellas Pharma, Bayer, Dendreon, Genentech/Roche, Merck, Seattle Genetics.

MTS: Consulting or Advisory Role - Janssen; Research Funding - AstraZeneca, Janssen, Madison Vaccines Inc., Pfizer, Roche, Zenith Epigenetics.

HHC: Research Funding - Astellas Medivation, Clovis Oncology, Color Foundation, Inovio Pharmaceuticals, Janssen, Sanofi. 
SPP: Honoraria - Prime Education; Travel, Accommodations, Expenses - Prime Education.

DWL: Consulting or Advisory Role - Astellas Pharma, Clovis Oncology, Dendreon; Research Funding - GenomeDx, Genomic Health, MDxHealth.

RBM: Research Funding - AstraZeneca, ESSA; Ferring, Janssen Oncology.

JLW: Speakers' Bureau - Foundation Health Partners Carroll Cancer Center, OncLive, Seattle Cancer Care Alliance; Research Funding - Altor BioScience, Merck, Nucleix; Other - Movember, UpToDate.

LND, TW, MST and FV-L have no relationships to disclose.

\section{REFERENCES}

[1] Humphrey PA, Moch H, Cubilla AL, Ulbright TM, Reuter VE. The 2016 WHO Classification of Tumours of the Urinary System and Male Genital Organs-Part B: Prostate and Bladder Tumours. European Urology. 2016;70(1): 106-19.

[2] Fox MD, Xiao L, Zhang M, Kamat AM, Siefker-Radtke A, Zhang L, Dinney CP, Czerniak B, Guo CC. Plasmacytoid Urothelial Carcinoma of the Urinary Bladder: A Clinicopathologic and Immunohistochemical Analysis of 49 Cases. American Journal of Clinical Pathology. 2017;147(5):5006.

[3] Keck B, Stoehr R, Wach S, Rogler A, Hofstaedter F, Lehmann J, Montironi R, Sibonye M, Fritsche HM, LopezBeltran A, Epstein JI, Wullich B, Hartmann A. The plasmacytoid carcinoma of the bladder-rare variant of aggressive urothelial carcinoma. International Journal of Cancer. 2011;129(2):346-54.

[4] Cockerill PA, Cheville JC, Boorjian SA, Blackburne A, Thapa P, Tarrell RF, Frank I. Outcomes Following Radical Cystectomy for Plasmacytoid Urothelial Carcinoma: Defining the Need for Improved Local Cancer Control. Urology. 2017;102:143-7.

[5] Kaimakliotis HZ, Monn MF, Cary KC, Pedrosa JA, Rice K, Masterson TA, Gardner TA, Hahn NM, Foster RS, Bihrle R, Cheng L, Koch MO. Plasmacytoid variant urothelial bladder cancer: is it time to update the treatment paradigm? Urologic Oncology. 2014;32(6):833-8.

[6] Ricardo-Gonzalez RR, Nguyen M, Gokden N, Sangoi AR, Presti JC, Jr., McKenney JK. Plasmacytoid carcinoma of the bladder: a urothelial carcinoma variant with a predilection for intraperitoneal spread. The Journal of Urology. 2012;187(3):852-5.

[7] Yin M, Joshi M, Meijer RP, Glantz M, Holder S, Harvey HA, Kaag M, Fransen van de Putte EE, Horenblas S, Drabick JJ. Neoadjuvant Chemotherapy for Muscle-Invasive Bladder Cancer: A Systematic Review and Two-Step Meta-Analysis. The Oncologist. 2016;21(6):708-15.

[8] Plimack ER, Hoffman-Censits JH, Viterbo R, Trabulsi EJ, Ross EA, Greenberg RE, Chen DY, Lallas CD, Wong YN, Lin J, Kutikov A, Dotan E, Brennan TA, Palma N, Dulaimi E, Mehrazin R, Boorjian SA, Kelly WK, Uzzo RG, Hudes GR. Accelerated methotrexate, vinblastine, doxorubicin, and cisplatin is safe, effective, and efficient neoadjuvant treatment for muscle-invasive bladder cancer: results of a multicenter phase II study with molecular correlates of response and toxicity. Journal of Clinical Oncology : Official Journal of the American Society of Clinical Oncology. 2014;32(18):1895-901.

[9] Dayyani F, Czerniak BA, Sircar K, Munsell MF, Millikan RE, Dinney CP, Siefker-Radtke AO. Plasmacytoid urothelial carcinoma, a chemosensitive cancer with poor prognosis, and peritoneal carcinomatosis. The Journal of Urology. 2013;189(5):1656-61.

[10] Hashemi-Sadraei N, Perrino CM, Monn MF, Bandali E, Cheng L, Idrees M, Bihrle R, Koch MO, Eble J, Kao C-S, Albany C, Pili R, Grignon DA, Kaimakliotis HZ. Plasmacytoid urothelial carcinoma: A clinicopathological study. Journal of Clinical Oncology. 2018;36(6_suppl):482.

[11] Teo MY, Al-Ahmadie H, Seier K, Ostrovnaya I, Regazzi AM, Dalbagni G, Bochner BH, Funt S, Iyer G, Bajorin DF, Rosenberg JE. Pre-operative chemotherapy (ctx) in plasmacytoid urothelial carcinoma (PUC). Journal of Clinical Oncology. 2018;36(6_suppl):522.

[12] Ericson KJ, Thomas L, Lee BH. Plasmacytoid variant urothelial carcinoma: Clinicopathologic outcomes and experience with neoadjuvant chemotherapy. Journal of Clinical Oncology. 2019;37(7_suppl):483.

[13] Li Q, Assel M, Benfante NE, Pietzak EJ, Herr HW, Donat M, Cha EK, Donahue TF, Bochner BH, Dalbagni G. The Impact of Plasmacytoid Variant Histology on the Survival of Patients with Urothelial Carcinoma of Bladder after Radical Cystectomy. European Urology Focus. 2019;5(1):104-8.

[14] Shariat SF, Karakiewicz PI, Palapattu GS, Lotan Y, Rogers CG, Amiel GE, Vazina A, Gupta A, Bastian PJ, Sagalowsky AI, Schoenberg MP, Lerner SP. Outcomes of radical cystectomy for transitional cell carcinoma of the bladder: a contemporary series from the Bladder Cancer Research Consortium. The Journal of Urology. 2006;176(6 Pt 1):2414-22; discussion 22.

[15] Hellenthal NJ, Hussain A, Andrews PE, Carpentier P, Castle E, Dasgupta P, Kaouk J, Khan S, Kibel A, Kim H, Manoharan M, Menon M, Mottrie A, Ornstein D, Palou J, Peabody J, Pruthi R, Richstone L, Schanne F, Stricker H, Thomas R, Wiklund P, Wilding G, Guru KA. Surgical margin status after robot assisted radical cystectomy: results from the International Robotic Cystectomy Consortium. The Journal of Urology. 2010;184(1):87-91.

[16] Power NE, Kassouf W, Bell D, Aprikian AG, Fradet Y, Lacombe L, Chin J, Izawa J, Estey E, Fairey A, Cagiannos I, Lattouf J-B, Drachenberg D, Rendon RA. Natural history of pT3-4 or node positive bladder cancer treated with radical cystectomy and no neoadjuvant chemotherapy in a contemporary North-American multi-institutional cohort. Canadian Urological Association journal=Journal de l'Association des urologues du Canada. 2012;6(6):E217E23.

[17] Perera M, McGrath S, Sengupta S, Crozier J, Bolton D, Lawrentschuk N. Pelvic lymph node dissection during radical cystectomy for muscle-invasive bladder cancer. Nature reviews Urology. 2018;15(11):686-92.

[18] Al-Ahmadie HA, Iyer G, Lee BH, Scott SN, Mehra R, Bagrodia A, Jordan EJ, Gao SP, Ramirez R, Cha EK, Desai NB, Zabor EC, Ostrovnaya I, Gopalan A, Chen YB, Fine SW, Tickoo SK, Gandhi A, Hreiki J, Viale A, Arcila ME, Dalbagni G, Rosenberg JE, Bochner BH, Bajorin DF, Berger MF, Reuter VE, Taylor BS, Solit DB. Frequent somatic 
CDH1 loss-of-function mutations in plasmacytoid variant bladder cancer. Nature Genetics. 2016;48(4):356-8.

[19] Perrino CM, Eble J, Kao CS, Whaley RD, Cheng L, Idrees M, Hashemi-Sadraei N, Monn MF, Kaimakliotis HZ, Bandali E, Grignon D. Plasmacytoid/diffuse urothelial carcinoma: a single institution immunohistochemical and molecular study of 69 patients. Human Pathology. 2019.
[20] Winters BR, De Sarkar N, Arora S, Bolouri H, Jana S, Vakar-Lopez F, Cheng HH, Schweizer MT, Yu EY, Grivas P, Lee JK, Kollath L, Holt SK, McFerrin L, Ha G, Nelson PS, Montgomery RB, Wright JL, Lam HM, Hsieh AC. Genomic distinctions between metastatic lower and upper tract urothelial carcinoma revealed through rapid autopsy. JCI Insight. 2019;5. 